and reduced to powder, they are given in drink to the patient. This preparation de. serres a place beside the oleum catullorum.

There was a peculiaricy of conformation in the bladder of this patient; it was bilobed and irregular, with fleshy columns on the right side. The stone was in the further cavity of the organ.

Case 2.-MIr. G__, aged 61 years, of nerrous habit, had been for a very long time under treatment for catarrh of the bladder; his medical attendant suspected that the affection depended upon the presence of a stone, particularly as the patient had formerly passed small gravel, but could not prevail on him to be sounded. As he was experiencing no relief, he betook himself to the use of one of those advertised remedies uhich cure all diseases. Some little amendment, coincident with the commencement of this self-prescribed treatment, inspired him with great confidence in its efficacy. Long and scrupulously did he persevere in the use of his pills, of which he has assured me he often consumed fourand-twenty in a day, and grew worse.

He came to me, recommended by MIr. Heath of Nottinghamshire, upon whom I operated for stone about two years ago. The introduction of the sound, and the exploration of the bladder, gave considerable pain; the existence of the stone was ascertained, and the irritability of the urethra was relieved by the use of a wax bougie, introduced for a few minutes daily, for a week. During this time a mild regimen was pre. scribed, with the tepid hip-bath and opiates. In this manner some amelioration of the srmptoms was obtained, and I was enabled to proceed with the operation. At the first sitting the patient manifested great anxiety lest the stone should prove too large for the grasp of the instrument, of this I promptly relieved him by telling him that it was now firmly fised in the crotchets of the forceps. The stone was about the size of a small walnut, and firiable. It was completely in fragments at the second sitting; and two more sittings sufficed for their entire removal. After the second sitting the irritability of the bladder ceased, indeed he might be said to suffer nothing more from his complaint; the urine, which had been very offensive, and loaded with mucus, had become clear; he ate and slept well, and seemed quite an altered man. The treatment of this case occupied only seventeen days from the commencement of the operation, and for the last ten of these he was able to walk, and attend to a little business. The gentlemen present at this operation were, AIr. Prater, Dr. Burke, Mr. Chapman, Mr. Ratray, Mr. Coleman, \&c.

7, Parliament-street, Westminster, January, 1833.

No. 489 .

\section{REMARKS ON TIE}

\section{MALIGNANT CIOLERA;}

ITS PATHOLOGY AND MEDICAL TREATMINRE.

$$
\begin{gathered}
\text { By T. G. Wrigar, M.D., M.R.C.S.L., } \\
\text { Siocliton-on.Tees. }
\end{gathered}
$$

Spasmodic cuolfina has happily, at length, so far abated its fatal ravages in this island, as to allow medical men leisure from the larass, fatigue, and excitement. into which they have been thrown for twelve months past, to look around them, and review what has been done br themselves and their contemporaries in investigating this dreadful malady; consequently. we may now hope for some more satisfactory exposition of it than has yet obtained amid the contention of opposing factions, and the inveterate warfare between contagionists and anticontagionists. In every quarter of Gıat Britain, carefully-observed facts have been quoted, and a profusion of monographs have been published on the subject; some excellent, and all showing an ardent desire on the part of the pro. fession to struggle successfully with this new pestilence. But, so far, no theory has received more than a partial assent, as accounting for the complex train of symptoms which accompany the disorder; nor has any principle of treatment been laid down, that furnishes a key to the operatiox of the various remedies which have been hitherto employed with apparent advantage. Both the nature and treatment of cholero are still involved in a mist of doubt and uncertainty: the most skilful research has failed to throw more light than that of probability on the one, nor has acute observa. tion and experience, in many millions of cases, been more successful in establishing the other.* Under these circumstances, it affords gratification to the writer of this article to remark, that a careful perusal of much (it were vain to say all) that has been written on the disease, a rather extensive. acquaintance with professional friends, who have had ample opportunities of observing and treating cholera, together with some personal experience, have tended strongly to confirm the opinions he adranced in

* It was stated in Thr LANCET, several months sinee, that twenty millions of persons have perished by cholera; but if we add up the statistical ac counts, we shall find this far below the reality, eighteen millions were carried off in the East Indies alone. Jonnés estimates tie lotal number of victums to the pest at wo or three times that amount; thesefore, averaging one-half of those attacked to have died, there must have been since 1817 , upwards of one hundred millions of cases of spasmodic cholera 
paper read to the Darlington Medical Society to pain) from the cerebro-spinal nelves, and on the 2nd of Iast $J$ anuary, and shortly their sensibility (to their own peculiar funcafterwards to the London University Medical tional stimulus) trom the ganglionic.

Society. The following observations con- 5. From the intimate anastomosis betain a more explicit detail of the views he tween every branch of the sympathetic was on the above occasions induced to take nerves, and a corresponding part of the of the pathology of spasmodic clsolera; he spinal or cerebral system, irritation of the is desirous to submit his ideas to the con- one class is speedily transferred to the sideration of his professional brethren, other; and the pneumogastric nerves, bethrough the medium of the valuable journal ing the pair most extensively connected in which they now appear.

Though the nature of cholera is yet im. perfectly understood, we are duily advancing toward a more correct knowledge of ir. The minutest analytical induction has been applied to the disease, and the result has been the accumulation of so voluminous a mass of valuable evidence on all points connected with it, as was never brought to bear upon one subject since medicine became a science. Analytical reasoning is the main-spring of medical argument; but after a disorder has been so thoroughly analysed, that the collection of facts becomes cumbrous on our hands, we may be excused in venturing to resort to synthesis. If ever such a mode be allowable,-though speculative hypotheses too often take the place of legitumate deduction, - it is surely the case now, in the malady under consideration.

In order, then, to illustrate more strilkingly the pathology of cholera, let us for a few moments dismiss the analysis of the disease entirely from our argument. Let us suppose some irritating, morbid, infectious. or epidemic influence, the effect of which shall be, through the medium of the bowels, on the ganglionic system of nerves. What, according to the principles of physiology and patbology, would be the train of symptoms produced by such an i:fluence?

In tracing the answer to this query, we must bear in mind the following propositions, which are so well established that they may be regarded as axioms.

1. The ganglionic or srmpathetic class of nerves, convey neither sensation nor mobility, but their action is referred solely to the functions of organic life, and chiefly to the various ofices connected with secretion.

2. The spinal nerves are the sources of feelisg and uotion; they supply all muscular structure, including both the voluntary and involuntary muscles; and hence,

3. The muscular coats of the bowels, as well as the muscular envelopes of the bron. chial tubes,* and the muscular fabric of the heart, are dependent on the motor branches of the cerebro-spinal system for their con tractility ; and, further,

4. The viscera derive their sensation (as

* See an inrrenious paper by Dr. Carson, junior (Lirerpool), in LAscer, April 7, 1832.

in such disorier.

6. Irritation of a nerve of motion pro. duces spasm in the muscular fibres supplied by that nerve. Irritation of a nerve of sensation causes pain, which is generally felt at the extremity of its ramification; but the only perceptible effect of irritation on a nerve of sense, or a nerve of organic life, is the diminution, or total loss of its func. tional power.

7 . Secretion is a process of life by which the blood is converted into certain healthy products ; and by affections of the sympathetic nerves, the secretions are altered in their nature. The only effect which we can conceive to be the result of a paralysis of those nerves, will be a conversion of the process of secretion into an exudation or elimination.

Some physiologists may hesitate to allow the third and fourth postulates; but I think it may be proved, that the functional sensibility of the involuntary muscles is derised from the sympathetic nerves; and that the impression received by them, which Joln Hunter aptly termed "the stimulus of necessity," is communicated to the motor branches, by which the muscular action is performed. Just as in a paroxysm of sneezing or coughing, the involuntury action of the muscles is owing to irritation of a nerve of sensation. In the eye, the phenomenon of winking, though performed by muscular neaves, is the result of a " stimulus of necessity," felt by the branches of the fifth pair in the conjunctiva. Numerous similar illustrations might be adduced. I decline in this place entering into a physiological discussion of the truth of the above postulates; but, being myself satisfied of their con restness, I am convinced of the sound. ness of the corollaries founded on them.

T'aking these data as the groundwork of our argument, we will endeavour to answer the problem advanced; viz., what would be the train of symptoms excited by a given infuence on the ganglionic nerves.

The immediate effect would be a disordered state of all the alvine secretions, in. ducing diarrhwa. Constipation could not be the result, as that would imply a tonic or astringent influence, which is contrary to the premises. The diarrhoa not being caused by irritating food, morbid biliary 
secretion, or drastic ingesta, but merely by the alvine secretions being more watery than natural, there would be no pain, because the nerves of sensation (wbich are sparin ly distributed over the intestines, with the exception of the rectum and the stomach, where only they are needed) are not acted on by any irritating matter within the bowels; but the motor branches distributed throughout the whole length of the alimentary canal are much more intimately concerned. From their universal anastomosis with the ganglionic nerves, there would arise a spasmodic action of the coats of the bowels and stomach; a violent and peculiar vomiting, with little or no concordant acion of the diaphragm or abdominal muscies, merely a contraction of the organ itself, accompanied by incessant purging, not with tenesmus or tormina, but the evacuations would be expelled without effort, and by a sort of pumping action of the bowel. This state having lasted a longer or shorter period, the fæcal matter of the bowels would be all carried off; but, the cause still remaining, the ganglionjc nerves would become paralysed. Elimina. tion would take the place of secretion into the intestines, and the watery parts of the blood be rapidly drained off; diarrhoea, or sickness, or boll, still continuing to carry ont of the body the evacuations, now become pale and serous, without smell or any of the characteristics of frecal matter.

Meantime the irritation would be extending along the whole of the sympathetic nerves. A change would take place in the secretions of all the glandular system. When the powers of organic life are weakened by ferer, bilious diarhoea, \&c., we observe that, in proportion as the secretion of the bowels is increased, the other secretions are diminished; so, in the case we are supposing, we should expect a total suspension of them. The bile would be no longer thrown into the duodenum; the pancreatic juice would fail to supply its portion, whatever that may be, to the alvine discharges; the urine would be suppressed; the detrusor muscles of the bladder would be in a state of spasmodic contraction, while the viscus itself was empty; the throat, tongue, and mouth, would be dry, and the skin livid and without perspiration; or, perhaps, a cold moisture migbt exude from the capillary pores; the eyes would be devoid of tears, and, from the experiments of Nona. Dupuy, * we might expect them to sink in their sockets and become dim. The irritation would also have extended to tho spinal column. Great pain and spasms would be liable to take place in all the mus.

* Quoted by Mr. H. Bell from the Journal de Médecine, tom. 37 , p. 343 . cles. The pneumogastric nerves would be especially affected: hence much disturbance in the circulation and respiration would ensue. This would be further increased by the blood having been deprived of great part of its fluid constituents; the circulation would be almost suspended, and the heat of the body, consequently, fall to a very low degree; the blood would hardly be propelled through the lungs ; of course the veins near the beart would be in a state of violent congestion; while, by means of the recurrent branch of the eighth pair, as well as from dryness of the larynx, the roice would become altered in tone and gradually lost. These symptoms would go on increasing with dreadful rapidity, and in a short time occasion denth, wilhout any disturbance of the mind, or any lesion of a single organ of sense; touch, perhaps, alone excepted.

These are all, and they are the only symptoms which we could calculate upon resulting from the cause we set ont by stating, and it is unnecessary to ask the reader to compare them with the symptoms of spasmodic cholera. Any one who had the painful office of visiting a patient labouring under that horrible malady, cannot fail to recognise, in this supposed case, an accurate picture of the real disease.

Let us now turn to the analysis of cbolera, and see how far it accords with our synthetical production. In all important particulars, post.mortem examinations, the most perfect of all our tests, confirm, to the letter, the correctness of the view here taken. It may, indeed, be objected, that dissection does not always show the ganglia to be unnatural in appearance, and a long list of cases might be quoted in support of this objection; but I cannot allow them to have much weight. We do not expect the scalpel uniformly, or eren generally, to expose distinct organic lesion in cases of paralysis and other disorders of the brain; and why should a more palpable alteration of structure be demanded in a similar affection of the ganglionic nerves, the pathology of which is less perfectly understood than that of the brain? If phy. siological signs of disordered function in this obscure system are detected, we must not refuse to be convinced they exist, though a visible lesion of structure is not always perceived. It is, however, at the same time to be recollected, that positive disorganization has frequently been noticed. The blood has been proved by $D r$. O'Shaughnessy, Dr. Ctanny, Mr. Rose, \&c. to be vitiated during all stages of cholera. Though the results of their experiments slightly differ, yet they all agree in the main point, viz. that the serous, i. e. the watery and saline parts of the blood, are

\& $\$ 2$ 
wanting. Upon this cause the above chemists suppose the disease to depend; but they seem to have stopped short in their patbological reasoning when just on the verge of important truth. How does this serous portion become wanting? If all the secretory vessels were in a state of health, there would be no possible mode of escape for the serum, except by the natural secretions. Unless, tberefore, wo suppose that disturbance of the secretory vessels precedes the loss of serum in the blood, we must assume, that an effect takes place without a cause. It has been demonstrated that the blood in all fevers undergoes some disorganization, which probably is the case also in cholera, independently of this fltering. Dr. Southwood Smith, Dr. Brown, and others, have spoken of the latter disease as a species of fever, and they undoubtedly have many points of resemblance; but till the pathological condition called fever is more correctly ascertained and understood, the comparison throws littie light upon the subject. The writer thinks that the state of the sympathetic nerves has not been sufficiently inquired into with regard to the latter class of diseases, fevers. $\mathrm{He}$ does not mean here to venture upon any hypothetical deductions as to their nature, but he wishes that the hint may be deemed worth attending to, by those who have opportunities for investigating it.

The view of cholera which is thus briefly sketched, occurred to the writer after a de. 1 berate consideration of the subject when in Paris eighteen months ago. It will be seen that the opinions advanced by several of the talented pathologists who have intermediately written on the disease, approach in some degree, though, essentially, they differ from that which he has ventured to suggest. The theory of $M r$. Hamilton Bell (one of the most plausible which bas been published) does not satisfactorily explain the occurrence of premonitory diarrhoa, nor indeed of diarrhwa at all, as a primary symptom : besides the division of the nervous system into three "powers" is neither so simple nor so explicit.as that which is so beautifully demonstrated by $M r$. Bell's distinguished uncle, Sir Charles. If Mr. Bell's theory were the true one, an in. jection of stimulant fluid into the veins, in order to rouse the action of the heart, or inhaling oxygen for a similar purpose, would, along with venesection, at once cure the disorder. The remark that saline injections seldom prove of permanent benefit, applies also to the views of $D r$. O'Shaughnessy, Mr. Annesley, and those who consider a morbid state of the blood as the primary source of cholera. The hypothesis of Dr. Kennedy and many others, that the seat of the malady is the spinal marrow, would lead us to expect pain, cramps, and spasms, or palsy in the extremities, as invariable and early symptoms preceding all others, which is certainly not the case. Neither can it be allowed that vomiting and pain in the stomach necessarily precetle diarrboa, as might be presumed from $M I$. Lizars having ascribed the proximate cause to inflammation of the eighth pair of nerves. If maligriant cholera were a " gatstro-enterite," as stated by Mr. Christie and many French writers, one would naturally infer that it might be subdued by those remedies which are found effectual in ordinary cholera morbus and bilious diarrhœen. That such is not the case in the malignant disease is evidenced by the various other modes of treatment which have been rea sorted to with advantage; and especially by the paradoxical fact, that a drastic cathartic so powerful as croton oil has the effect, in spasmodic cholera, of checking the diarrhea and substituting mild evacuations of fecal matter for the " rice-water" discharges. Unless any hypothesis of the late unfortu. nate Professor Delpech be similar to the one at present under discussion, there is ro view which has fallen under the observation of the writer that is free from these various objections, or which will ex. plain the singular anomaly just alluded to, except that which he is endeavouring to substantiate.

It is immaterial to the preceding argument whether cholera be epidemic, infectious, or contagious. In the MS. before alluded to, similar opinions are advocated to those now generally prevalent among medical men who have seen much of the disease, viz., that it is usually epidemic, and occasionally but rarely infectious. The writer has also seen well-marked cases of malignant cholera, both endemic and sporadic. The atmosphere appears to have little share in producing or propagating the disorder, unless, as Sir H. Davy hinted, the air has " something more than its pon. derable elements." Has the earth some influence on the powers of life which philosophy has not yet detected?

Let it not be said, however, that all this is speculative theory; for the great object of this paper is to elicit practical benefit in the treatment of cholera. So far various plans have been adopted, but few of them have been derived from any fixed principle. Most of them are recommended by ex. perience alone; and experience, alas! has failed to speak much in favour of the most successful ones. In a large proportion of cases, where the patients' constitutions were very unhealthy, and the disease had got a firm loold, the most consummate medical skill appears to have been of little avail; but if those methods of treatment 
which have been found most serviceable can all be reduced to one and the same principle, and that principle be found to agree with the most plausible theory of the patholosy of cholera, it is fair to infer, that by modifying and adapting our remedies to fulfil the indications thus pointed out, we may arrive at the most beneficial practice which the nature of the case admits of.

As paralysis of the ganglionic nerves is a pathological condition which has seldom, perlaps, presented itself to the mind of the therapeutist, we can only draw the indications of treatment fiom analogy. In ordinary loss of power of a nerve of sense (or, indeed, of any nerve), it is usual to employ stimulants, and hence, in our view of cholera, we should be induced, a priori, to administer the onl 5 stimulants we know of, affecting the abdominal part of the ganglionic system, viz, aperients or purgatives, combined with calomel, perbaps adding a little opium at first, to allay the irritation of the spinal nerves. Let the following plans of treatment, decidedly the most successful on record, be my answer to the question :-

Mr. Searle's exhibition of large quantities of table salt.

Mr. Montgomery's employment of calomel, capsicum, and opium.

Dr. Kennedy's plan by bleeding and calomel.

Dr. Hacket (of Trinidad), and Dr. Tegart's recommendation of croton oil ; a moilified use of which, I have been informed, was successfully adopted during the late virulent irruption of the malady at Stockton.

Dr. Ayre's frequently-repeated doses of calomel, a plan which $M r$. H. Thomas, of Sheffield, acquaints me, has been attended with marked success in that town.

Mr. M'Intyre's (of Newcastle) treatment by castor oil and aromatics, with calomel, small doses of opium, and copious enemas of warm gruel and turpentine, which was so successful in that gentleman's extensive colliery practice, that I am authorised, on his own information, to state, he and his assistants did not lose more than about 80 out of 700 cases.

With most of the above modes of treatment, early bleeding is insisted on. It is a point of great importance mechanically to relieve the loaded veins of a portion of their dark contents. And here it should be remembered, that a small pulse no more contra indicates that practice in the malignant disease, than it does in enteritis; but for a similar reason in both cases (so far as the cause of the weak pulse is concerned) ur gently calls for venesection. More or less diffusible stimulus, in order to rouse the faltering powers of life, is also generally recommended in spasmodic cholera.

A word on the saline injections into the veins. If the foregoing sketch be correct, it explains the curious train of effects following this novel course of practice. Fluid. ity is restored to the blood; but it is like water poured into a sieve,-the liquid soon runs off by the bowels, which are not at all acted on by the venous injection, and the patient too often sinks from a state of appa rent revival into one of fatal relapse. As a measure of the success which has attended this plan, the writer has collected from various periodical journals, reports of 105 cases, in which fluid of various kinds, generally in solution of salts, had been injected into the veins of cholera patients. He has arranged them in a tabular form, showing the age of the individuals, the number of hours which elapsed from the time of attack before injection was resorted to, the quantity injected in a given time, and the event. The following is a summary of his list.

\begin{tabular}{|c|c|c|c|c|c|c|c|c|c|c|}
\hline No. & Hours ill. & & Injected. & Cured. & Con & rales & cent. & Under 1 & reatmt. & Dier? \\
\hline 15 & $\ldots 4$ to 12 & $\ldots$ & $=24$ to 305 & $\ldots \ldots 1$ & $\ldots$ & 2 & $\ldots$ & - & $\ldots \ldots$ & 12 \\
\hline 13 & $\ldots \ldots 12$ to 27 & $\ldots$ & $\xi 23$ to 292 & $\ldots 3$ & $\ldots$ & 4 & $\ldots$ & - & $\ldots \ldots$ & . 6 \\
\hline 77 & .... unknown & $\ldots$ & 314 to 640 & $\ldots \ldots 1$ & $\ldots$ & 1 & $\cdots$ & 15 & $\ldots \ldots$ & . 60 \\
\hline 105 & & & 31 & 5 & & $i$ & & 15 & & 78 \\
\hline
\end{tabular}

The method has not often been success- is practically worthy of attention. The ful, and seems now pretty generally aban- writer hopes what has been said is sufdoned; but, combined with the administra- ficient for that purpose; and, though not so tion of aperients, calomel, and other reme- sanguine as to believe that he has solred dial measures, it might, perhaps, be of great all the intricacies of the subject, or that the advantage. It has been by relying on the sketch he has given is free from objections, injections as a permanent instead of a tem- he thinks it is calculated to remove some porary relief, that much of the failure of difficulties; hence, he shall esteem bimself that system is probably to be attributed.

A mass of further authorities might be adduced, to convince the reader that the view of cholera which is here briefly taken,

fortunate if his remarks advance us ane step farther in the elucidation of this ingportant department of medical science. Stockton-on-Tees, January, 1833. 